\title{
Influence of fluor salts, hormone replacement therapy and calcitonin on the concentration of insulin-like growth factor (IGF)-I, IGF-II and transforming growth factor- $\beta 1$ in human iliac crest bone matrix from patients with primary osteoporosis
}

\author{
C E Pepene, T Seck ${ }^{1}$, I Diel ${ }^{2}$, H W Minne ${ }^{3}$, R Ziegler and J Pfeilschifter ${ }^{1}$ \\ Department of Internal Medicine I, University of Heidelberg, Heidelberg, Germany, ${ }^{1}$ Department of Medicine, BG-Kliniken Bergmannsheil, \\ University of Bochum, Bochum, Germany, ${ }^{2}$ Department of Gynecology, University of Heidelberg, Heidelberg, Germany and ${ }^{3}$ Klinik 'Der Fürstenhof', \\ Bad Pyrmont, Germany
}

(Correspondence should be addressed to C E Pepene who is now at the Clinic of Endocrinology, Louis Pasteur Street 3-5, 3400 Cluj-Napoca, Romania; Email: carmenpepene@yahoo.com)

\begin{abstract}
Objective: Data from cell culture experiments suggest that local growth factors (GFs) may mediate the effects of estrogens, calcitonin or fluor ions on the skeleton. To assess the in vivo relevance of the in vitro reports, the effect of fluor salts, hormone replacement therapy (HRT) and calcitonin on the concentrations of IGF-I, IGF-II and transforming growth factor (TGF)- $\beta 1$ in bone matrix extracts from osteoporotic patients was evaluated.

Design: Iliac crest bone biopsies were obtained from 170 patients (76 men and 94 women) with primary osteoporosis aged $55.5 \pm 0.8$ years.

Methods: Bone matrix extraction was performed based on a guanidine-HCl/ethylendiamine-tetraacetic acid method.

Results: In comparison with age- and body mass index (BMI)-matched controls, no influence of longterm therapy with fluor ions $(n=41)$ or calcitonin $(n=16)$ on the bone matrix concentration of GFs was noticed. Postmenopausal women with osteoporosis on HRT $(n=39)$ had lower skeletal IGF-I but not IGF-II levels as compared with age- and BMI-matched non-users. However, the lower rate of bone turnover in women with HRT may account for this difference, since the significance was lost after adjustment for alkaline phosphatase. Likewise, a tendency for lower TGF- $\beta 1$ levels was observed in HRT users as compared with non-users but was lost after adjustment for bone turnover. None of the therapies influenced the serum levels of GFs when patients receiving continuous therapy for at least 1 year before bone biopsy were considered.

Conclusions: Our data suggest no direct effect of fluor therapy on skeletal GFs levels. At the concentrations used, neither HRT nor calcitonin appeared to exert any significant influence on serum or bone matrix GF levels.
\end{abstract}

European Journal of Endocrinology 150 81-91

\section{Introduction}

Insulin-like growth factors (IGFs) and transforming growth factor (TGF)- $\beta 1$ are major regulators of human bone remodeling. The peptides promote osteoblast $(1-4)$ and osteoclast activity (5) and may act as coupling factors of bone resorption to subsequent bone formation (6). In vitro experiments have demonstrated interactions between these skeletal growth factors and hormones or substances presently used as therapeutic agents in metabolic bone diseases, such as estrogens, calcitonin or fluoride. Fluoride has been shown to potentiate the mitogenic effect of IGF-I on osteoblastic cells in vitro (7), thereby increasing osteogenic cell proliferation. In rodent and human osteoblastic systems, estradiol enhances IGF-I expression by osteoblasts $(8,9)$, suggesting that, in part, the effects of estrogens on bone cells may be mediated by IGF-I. However, in these experiments, estradiol was able to increase IGF-I transcripts but not polypeptide levels. In addition, a stimulatory effect of estrogens on TGF- $\beta 1$ production and activity $(10,11)$ has been demonstrated in cell culture systems. Recent research has shown that calcitonin is able to stimulate the production of IGF-I and IGF-II in human osteoblastic cells (12). Based on these results, we hypothesized that skeletal growth factors may, in part, mediate the positive effects of these agents on bone. On the other hand, apart from the production of 
growth factors by bone cells, the in vivo concentration of IGF-I, IGF-II and TGF- $\beta 1$ in bone matrix may depend to a great extent on several parameters, such as age, body mass index (BMI) $(13,14)$, bone turnover or bone mass. Whereas the concentration of TGF- $\beta 1$ in the human skeleton appeared to be related to bone turnover but not to bone mass (15), the skeletal content of IGF-I was positively associated with both bone turnover (16-18) and bone volume (17). Thus, it remains unclear whether in vitro osteogenic actions of estrogens and other agents have a biological relevance in vivo. In the present study, we aimed to establish the effects of fluor ions, hormone replacement therapy (HRT) and calcitonin on the bone matrix concentration of IGF-I, IGF-II and TGF- $\beta 1$ in subjects with primary osteoporosis.

\section{Subjects and methods}

\section{Bone samples}

Iliac crest bone biopsies were obtained from 170 patients with osteoporosis (76 men and 94 women) who were inpatients at the Department of Endocrinology of the University of Heidelberg and the Clinic of Metabolic Diseases, Bad Pyrmont, Germany. All patients gave informed consent and the study was conducted with the approval of the ethics committee on clinical investigations at the University of Heidelberg Medical Center.

Osteoporosis was considered as a bone mineral density (BMD) $\mathrm{T}$ score at the lumbar spine (L2-L4) and/or the hip $=$ or $<-2.5$ s.D.

Exclusion criteria were corticosteroid therapy for more than 3 months in the past 3 years, hyperparathyroidism, overt hyperthyroidism, evidence of systemic mastocytosis, plasmocytoma or renal osteopathy, anticonvulsant therapy, lactose intolerance, malabsorption and histological signs of osteomalacia. Donor-specific data were recorded with respect to age, BMI, physical activity, current smoking, daily alcohol intake, and calcium and vitamin D supplement intake. Of the 170 patients, in 107 patients (39 men and 68 women) osteoporosis therapy had been administered for at least 3 months in the year before bone biopsy. Fortyfive postmenopausal women had received HRT, 67 patients (32 men and 35 women) had received therapy with fluor ions and 16 patients ( 10 men and six women) had used calcitonin as a therapeutic agent. Calcitonin was given either subcutaneosly (100-200 IU/day) or intranasally (200 IU/day). About $6.6 \%$ of the male and $26.6 \%$ of the female patients had received more than one of these drugs for at least 3 months in the year before bone biopsy. Apart from calcium and vitamin D supplement administration, osteoporosis was not treated in the remaining 37 men and 26 women respectively.
Bone biopsies were obtained by horizontal approach, were about $5-7 \mathrm{~mm}$ in diameter and had an average length of $1.5-2 \mathrm{~cm}$.

Blood samples were obtained in a subset of 135 patients (61 men and 74 women) for the measurement of growth factors in serum. After collection, both bone and serum samples were stored at $-80^{\circ} \mathrm{C}$ until further analysis.

\section{BMD measurements}

Using dual X-ray absorptiometry (Hologic 1000, Hologic 2000, Hologic Inc., Waltham, MA, USA and DPXL, Lunar, Madison, WI, USA), BMD was determined as bone mineral content per $\mathrm{cm}^{2}$ of the lumbar spine (L2-L4) and the left hip. Quality control was ensured by daily calibration using the phantom provided by the manufacturer. Cross-calibration of instruments, allowing intercomparison of results obtained with machines from different manufacturers was performed using the European Spine Phantom prototype, according to the European Concerted Medical Action protocol (19).

\section{Bone matrix extraction}

Bone samples were cleaned mechanically and cancellous bone was separated from cortical bone using a dissection microscope. Cancellous bone fragments $(0.1-0.5 \mathrm{~cm}$ diameter) were washed repeatedly in cold distilled water until the washes were free of blood and then defatted in di-isopropylether $\left(4^{\circ} \mathrm{C}\right)$ for $2 \mathrm{~h}$. The defatted bone samples were ground for $10 \mathrm{~min}$ into small particles $(40-60 \mu \mathrm{m})$ in a liquid nitrogen-cooled freezer mill (Raetsch, Haan, Germany), using a wolfram-carbide ball. The yield of mineralized bone powder was then washed-out with cold distilled water, centrifuged and lyophilized overnight. Duplicate bone matrix extractions were performed for each bone sample, as previously described (20). The results from the two measurements were then averaged. Lyophilized bone powder $(15 \mathrm{mg})$ was placed in a microcentrifuge tube (Twist-Lock; Eppendorf, Hamburg, Germany) and $1.7 \mathrm{ml}$ extraction solution added. In $14.7 \%$ of bone samples less than $15 \mathrm{mg}$ bone powder was available for extraction.

Spectrapor 3-dialysis tubing (3.5 kDa cut-off; Spectrum Medical Industries, Houston, TX, USA) was placed over the tube opening and secured by a melted-out $(3-4 \mathrm{~mm})$ tube cap. The tubes were inverted and firmly fixed upside down in a circular styropor rack floating on top of the extraction solution ( $2500 \mathrm{ml}$ for 60 tubes). Extraction was achieved by passive dialysis against $0.05 \mathrm{~mol} / \mathrm{l}$ tetrasodium EDTA (Serva, Heidelberg, Germany), $4 \mathrm{~mol} / \mathrm{l}$ guanidine- $\mathrm{HCl}$ (Sigma, Deisenhofen, Germany), $30 \mathrm{mmol} / \mathrm{l}$ Tris (Merck, Darmstadt, Germany) and $1 \mathrm{mg} / \mathrm{ml}$ bovine serum albumin (Sigma; radioimmunoassay grade) at $\mathrm{pH}$ 7.4. The following protease inhibitors were added 
to the extraction solution: $5 \mathrm{mmol} / \mathrm{l}$ benzamidine $-\mathrm{HCl}$, $1 \mathrm{mmol} / \mathrm{l}$ phenylmethylsulfonyl fluoride and $0.1 \mathrm{~mol} / \mathrm{l}$ $\epsilon$-aminocaproic acid (Sigma). Dialysis was carried out at $4{ }^{\circ} \mathrm{C}$ under constant stirring for $24 \mathrm{~h}$. Consecutively, the samples were redialyzed for $72 \mathrm{~h}$ against $10 \mathrm{mmol} / \mathrm{l}$ acetic acid ( $\mathrm{pH} 2.8)$. The dialysis medium $(2500 \mathrm{ml}$ for 60 tubes) was replaced every $24 \mathrm{~h}$. After centrifugation at $10000 \boldsymbol{g}$ for $5 \mathrm{~min}$, the supernatant extracts were recovered and stored at $-80^{\circ} \mathrm{C}$ until assayed for growth factors concentration. Intra- and inter-extraction coefficients of variance were less than $9 \%$ and $15 \%$ respectively for all growth factors.

Normalized growth factor concentration was calculated per mg mineralized bone or per mg dry weight of the non-extractable bone matrix, which reflects the amount of collagenous bone matrix appropriately. The residual bone matrix after extraction was therefore twice resuspended in distilled water, centrifuged at $10000 \boldsymbol{g}$ for $2 \mathrm{~min}$, lyophilized and weighed. Normalized indices for IGF-I $(r=0.51, P<0.0001, n=142)$, IGF-II $(r=0.28, P=0.001, n=129)$ and TGF- $\beta 1(r=0.74$, $P<0.0001, n=142$ ) were highly correlated.

\section{Measurements of IGF-I, IGF-II and IGF-binding protein (IGFBP)-3 in bone matrix extracts and/or serum}

IGF-I was quantified in bone matrix extracts and in serum as described previously (17), using IGF-I (Mediagnost, Tübingen, Germany) and recombinant human IGF-I (GroPeP, Adelaide, Australia) as a tracer and standard $(20,21)$. IGFBP artifacts were avoided by initial dissociation of IGF from the binding proteins present using an acidic buffer. Inducing binding protein saturation through the addition of excess IGF-II (Mediagnost) then blocked reassociation. Serum and extraction samples were diluted by a factor of 50 and 5 respectively. This assay method has been extensively validated and shows good correlation with measurements obtained by acidic size-exclusion chromatography (22). Previous experiments had shown that with this procedure unlabeled IGF-I is completely recovered after preincubation with the extracts and that there is no change in the measurement of endogenous IGF-I in the presence of up to $10 \mathrm{ng} / \mathrm{ml}$ IGFBP-3 (17). The sensitivity of the IGF-I radioimmunoassay was $0.002 \mathrm{ng} /$ tube $(0.02 \mathrm{ng} / \mathrm{ml})$. The interassay variance of measurement for IGF-I was 7\% and IGF-II cross-reactivity was below $0.05 \%$. Assay conditions for IGF-II were similar to those described for IGF-I, except that IGF-I excess was used to avoid artifacts induced by IGFBPs $(22,23)$. The IGF-II assay had an interassay precision of $6.5 \%$.

IGFBP-3 was measured in serum using a commercial enzyme-linked immunosorbent assay from Biomerieux, Germany. The sensitivity of the assay is given as $0.04 \mathrm{ng} / \mathrm{ml}$. The interassay coefficient of variation was $9.9 \%$.

\section{Measurement of TGF- $\beta 1$ in bone matrix extracts and/or serum}

Immunoreactive TGF- $\beta 1$ in bone matrix extracts and serum was determined by specific enzyme-linked immunoassay (Genzyme, Cambridge, MA, USA), using a direct-labeled HRP-conjugated polyclonal antibody to TGF- $\beta 1$ and natural human TGF- $\beta 1$ as a standard. Activation of TGF- $\beta 1$ prior to its measurement was performed by acidification. Serum and extraction samples were diluted by a factor of 50 and 4 respectively. We have validated the assay by showing good parallelism of the dilution curves of bone extracts and TGF- $\beta 1$ standard curves. The assay sensitivity was $0.05 \mathrm{ng} / \mathrm{ml}$. The interassay variance of measurements for TGF- $\beta 1$ was $7.5 \%$.

\section{Statistical analysis}

Results are expressed as means \pm S.E.M. Data were analyzed using the Statistical Analysis System software program (SAS Institute, Cary, NC, USA). $P$ values $<0.05$ were considered statistically significant. Pearson's simple and partial correlation coefficients were used to describe the associations between two continuous variables. The general linear model procedure was used to analyze differences between groups. In order to adjust for differences in life-style factors or therapy in multivariate analyses, the clinical data were transformed in categorical variables.

\section{Results}

Baseline characteristics and BMD values of 170 patients with osteoporosis (76 men and 94 women), aged 55.5 \pm 0.8 years (range 27-88 years) are presented in Table 1. As shown, in our study group, men were significantly younger and more likely to drink alcohol than were the women, whereas no gender differences were observed with respect to physical activity (Table 1). The mean length of therapy with fluor salts, HRT and calcitonin in the past $1-3$ years before biopsy in subjects with primary osteoporosis in whom the effect of anti-osteoporotic agents on skeletal growth factors was analyzed is shown in Table 2.

\section{Effects of therapy with fluor ions on bone matrix and serum levels of IGF-I, IGF-II and TGF- $\beta 1$ in patients with primary osteoporosis}

To evaluate the influence of therapy with fluor ions on the bone matrix content of IGF-I, IGF-II and TGF- $\beta 1$, 41 patients (26 men and 15 women) receiving fluor therapy (sodium fluoride, monofluorphosphate) were compared with 47 age- and BMI-matched patients (29 men and 18 women). Apart from calcium and vitamin D supplements that had been administered to patients from both groups, patients in the control 
Table 1 Baseline characteristics and cross-calibrated lumbar spine and hip BMD mean values in 170 patients with primary osteoporosis at the time of iliac crest biopsy. When all patients were considered, BMD measurements were available, from left to right, for the lumbar spine in 146, 67 and 79 of patients and for the hip in 138,61 and 77 of patients respectively $\left({ }^{a} P=0.02,{ }^{b} P=0.0002\right)$. In fluor salt-treated patients' BMD measurements were available, from left to right, for the lumbar spine in 35,23 and 12 of patients and for the hip in 32, 23 and 12 of patients respectively. In women taking HRT, BMD was assessed at the lumbar spine in 37 and at the hip in 31 . BMD was available in calcitonin-treated subjects, from left to right in 13,9 and 4 patients for the lumbar spine and the hip respectively. Data are expressed as means \pm S.E.M.

\begin{tabular}{|c|c|c|c|c|c|c|c|c|c|c|}
\hline & \multicolumn{3}{|c|}{ All } & \multicolumn{3}{|c|}{ Fluor therapy } & \multirow{2}{*}{$\begin{array}{c}\text { HRT } \\
\text { Women }\end{array}$} & \multicolumn{3}{|c|}{ Calcitonin therapy } \\
\hline & All & Men & Women & All & Men & Women & & All & Men & Women \\
\hline Number & 170 & 76 & 94 & 41 & 26 & 15 & 39 & 16 & 10 & 6 \\
\hline Age (years) & $55.5 \pm 0.8$ & $51.9 \pm 0.9$ & $58.4 \pm 1.3^{b}$ & $55.5 \pm 1.6$ & $54.8 \pm 1.2$ & $56.7 \pm 3.6$ & $56.2 \pm 1.0$ & $59.0 \pm 3.2$ & $51.5 \pm 3.1$ & $71.5 \pm 2.6$ \\
\hline $\begin{array}{l}\mathrm{BMI}\left(\mathrm{kg} / \mathrm{m}^{2}\right) \\
\operatorname{BMD}\left(\mathrm{g} / \mathrm{cm}^{2}\right)\end{array}$ & $23.9 \pm 0.2$ & $24.4 \pm 0.3$ & $23.5 \pm 0.3$ & $24.6 \pm 0.4$ & $24.9 \pm 0.6$ & $24.0 \pm 0.7$ & $23.7 \pm 0.5$ & $22.6 \pm 0.5$ & $22.8 \pm 0.7$ & $22.5 \pm 0.8$ \\
\hline $\mathrm{L}_{2}-\mathrm{L}_{4}$ & $0.72 \pm 0.01$ & $0.74 \pm 0.01$ & $0.70 \pm 0.01$ & $0.73 \pm 0.01$ & $0.75 \pm 0.02$ & $0.71 \pm 0.03$ & $0.70 \pm 0.02$ & $0.75 \pm 0.03$ & $0.76 \pm 0.04$ & $0.72 \pm 0.04$ \\
\hline FN & $0.60 \pm 0.01$ & $0.65 \pm 0.01$ & $0.52 \pm 0.03$ & $0.60 \pm 0.01$ & $0.62 \pm 0.02$ & $0.56 \pm 0.02$ & $0.52 \pm 0.05$ & $0.64 \pm 0.05$ & $6.72 \pm 0.03$ & $0.47 \pm 0.07$ \\
\hline WT & $0.44 \pm 0.01$ & $0.46 \pm 0.01$ & $0.40 \pm 0.02$ & $0.42 \pm 0.01$ & $0.42 \pm 0.02$ & $0.41 \pm 0.03$ & $0.40 \pm 0.06$ & $0.45 \pm 0.04$ & $0.52 \pm 0.05$ & $0.28 \pm 0.05$ \\
\hline $\mathrm{L}_{2}-\mathrm{L}_{4}$ & $-3.38 \pm 0.08$ & $-3.38 \pm 0.12$ & $-3.39 \pm 0.11$ & $-3.31 \pm 0.17$ & $-3.32 \pm 0.21$ & $-3.30 \pm 0.31$ & $-3.39 \pm 0.17$ & $-3.22 \pm 0.30$ & $-3.19 \pm 0.41$ & $-3.26 \pm 0.35$ \\
\hline & $-3.39 \pm 0.18$ & $-2.95 \pm 0.12$ & $-3.73 \pm 0.30$ & $-3.25 \pm 0.15$ & $-3.18 \pm 0.18$ & $-3.36 \pm 0.28$ & $-3.76 \pm 0.54$ & $-2.92 \pm 0.37$ & $-2.35 \pm 0.32$ & $-4.18 \pm 0.66$ \\
\hline WT & $-3.34 \pm 0.11$ & $-3.05 \pm 0.12$ & $-3.57 \pm 0.18$ & $-3.43 \pm 0.16$ & $-3.40 \pm 0.16$ & $-3.4 \pm 0.33$ & $-3.64 \pm 0.37$ & $-3.22 \pm 0.44$ & $-2.58 \pm 0.46$ & $-4.65 \pm 0.58$ \\
\hline $\mathrm{AP}(\mathrm{U} / \mathrm{l})$ & $107 \pm 3$ & $107 \pm 5$ & $106 \pm 4$ & $106 \pm 7$ & $94 \pm 7$ & $127 \pm 12^{a}$ & $94 \pm 6$ & $123 \pm 13$ & $108 \pm 12$ & $149 \pm 23$ \\
\hline$\%$ VF & 46.4 & 40.7 & 51.0 & 34.1 & 34.6 & 33.3 & 41.0 & 62.5 & 50.0 & 83.3 \\
\hline$\%$ alcohol & 4.1 & $7.9^{a}$ & 1.1 & 4.8 & 7.6 & - & - & 12.5 & 20 & - \\
\hline$\%$ physical activity & 32.3 & 22.3 & 40.4 & 24.3 & 19.2 & 33.3 & 35.8 & 37.5 & 30 & 50 \\
\hline
\end{tabular}

$\mathrm{L}_{2}-\mathrm{L}_{4}=$ Lumbar spine; $\mathrm{FN}=$ femoral neck; $\mathrm{WT}=$ Ward's triangle; $\mathrm{AP}=$ alkaline phosphatase; $\mathrm{VF}=$ vertebral fractures 
Table 2 Mean length of therapy with fluor salts, HRT and calcitonin in subjects with primary osteoporosis. Data are expressed as means \pm S.E.M.; every patient had received at least 3 months of therapy during the last year before bone biopsy.

\begin{tabular}{|c|c|c|c|c|c|c|c|}
\hline & \multicolumn{3}{|c|}{ Fluoride } & \multirow{2}{*}{$\frac{\text { HRT }}{\text { Women }}$} & \multicolumn{3}{|c|}{ Calcitonin } \\
\hline & All & Men & Women & & All & Men & Women \\
\hline Number & 41 & 26 & 15 & 39 & 16 & 10 & 6 \\
\hline $\begin{array}{l}\text { Mean length of therapy in the past year } \\
\text { before biopsy (month) }\end{array}$ & $10.0 \pm 0.4$ & $9.9 \pm 0.5$ & $10.1 \pm 0.7$ & $10.8 \pm 0.4$ & $9.5 \pm 0.8$ & $9.9 \pm 0.9$ & $8.8 \pm 1.5$ \\
\hline $\begin{array}{l}\text { Mean length of therapy in the past } 3 \text { years } \\
\text { before biopsy (month) }\end{array}$ & $18.9 \pm 1.8$ & $19.2 \pm 2.3$ & $18.3 \pm 3.2$ & $26.9 \pm 2.0$ & $17.3 \pm 3.1$ & $17.9 \pm 4.1$ & $16.5 \pm 5.0$ \\
\hline
\end{tabular}

group did not receive any specific therapy for osteoporosis. No differences in the bone matrix concentration of IGF-I or IGF-II between groups were detected by simple analysis or after adjustment for life-style factors and calcium and vitamin D intake, in either men or women (Fig. 1A and B). In addition, fluor therapy had no influence on the concentration of TGF- $\beta 1$ in the bone matrix of patients with primary osteoporosis (Fig. 1C). Likewise, restriction of the analysis to the group of patients with osteoporosis using fluor therapy continuously for at least 12 months before iliac crest biopsy $(n=25)$ showed no differences in the concentration of bone matrix growth factors in comparison with age- and BMI-matched controls $(n=43)$ (data not shown). There was no evidence for an association between the length of therapy and the cancellous bone level of the growth factors, as detected by simple regression analysis (men: IGF-I: $n=25, r=0.31, P=0.12$; IGF-II: $n=21, r=0.06$, $P=0.78 ; \quad$ TGF- $\beta 1: \quad n=17, \quad r=-0.03, \quad P=0.86$; women: IGF-I: $n=13, r=-0.23, P=0.43$; IGF-II: $n=13, \quad r=-0.35, \quad P=0.23 ; \quad$ TGF- $\beta 1: \quad n=14$, $r=0.19, P=0.51)$. Likewise, stratification of data by length of therapy (i.e. 3-6 months of treatment, 6-9 months, 9-12 months and $\geq 12$ months of therapy) revealed no differences between groups (data not shown). In addition, the total amount of fluor ions received by patients during therapy had no influence on the skeletal concentrations of IGF-I, IGFII or TGF- $\beta 1$ (data not shown).

We further analyzed the effect of therapy with fluor salts on the serum concentration of growth factors between the group of patients to whom fluor ions had been administered for at least 12 months before biopsy (15 men and 10 women) and age- and BMImatched non-users (30 men and 15 women). Fluor salt users had levels of serum IGF-I and IGF-II comparable with non-users in either men or women (Fig. 1D and $\mathrm{E}$ ), regardless of whether adjustment for serum IGFBP-3 levels was performed or not. In addition, we were not able to detect differences in the concentration of serum TGF- $\beta 1$ between fluor ion users and non-users, in either men or women with osteoporosis (Fig. 1F).

\section{The effects of HRT on bone matrix and serum levels of IGF-I, IGF-II and TGF- $\beta 1$ in postmenopausal women with osteoporosis}

The effect of HRT on the concentration of IGF-I, IGF-II and TGF- $\beta 1$ in iliac crest bone matrix was assessed in 39 postmenopausal women with osteoporosis, aged $56.5 \pm 1.0$ years. Patients had received HRT for $26.9 \pm 2.0$ months in the past 3 years (Table 2). A group of 13 age- and BMI-matched postmenopausal women without HRT diagnosed with osteoporosis were considered as controls. Based on our observation that fluor therapy had no significant impact on the trabecular IGF or TGF- $\beta 1$ content, women receiving fluor therapy $(n=15)$ besides HRT were not excluded from analysis but adjustment for fluor salt treatment was performed. As expected, due to the decrease in the rate of bone turnover induced by HRT, lower levels of serum total alkaline phosphatase (AP) levels were noticed in women with HRT in comparison to women without HRT (HRT users 123.3 $\pm 9.8 \mathrm{IU} / \mathrm{l}$ vs non-users $94.4 \pm 5.6 \mathrm{IU} / \mathrm{l}, P=0.01)$. After adjustment for lifestyle factors, fluor therapy, and calcium and vitamin D intake, skeletal IGF-I but not IGF-II levels were significantly decreased in women with HRT as compared with women without HRT (Fig. 2A and B). However, this was, presumably, due to the effects of HRT on bone turnover, since the difference was no more significant after adjustment of data for serum AP concentrations. In contrast, HRT had no influence on bone matrix IGF-II levels. There was a tendency for lower bone matrix TGF- $\beta 1$ levels in women with HRT as compared with women without HRT $(P=0.07)$, but this was lost after adjustment for serum AP levels (Fig. 2C). Similar results were obtained between the group of postmenopausal women with osteoporosis who had used HRT continuously for at least 12 months before bone biopsy $(n=32)$ and age- and BMI-matched controls without HRT (data not shown). There was no evidence for an association between the skeletal levels of IGF-I or IGF-II and the length of therapy in HRT users (IGF-I: $n=38, r=-0.19, P=0.23$; IGF-II: $n=38$, $r=-0.09, P=0.56)$. In our group of postmenopausal patients, a weak inverse linear correlation between 
A

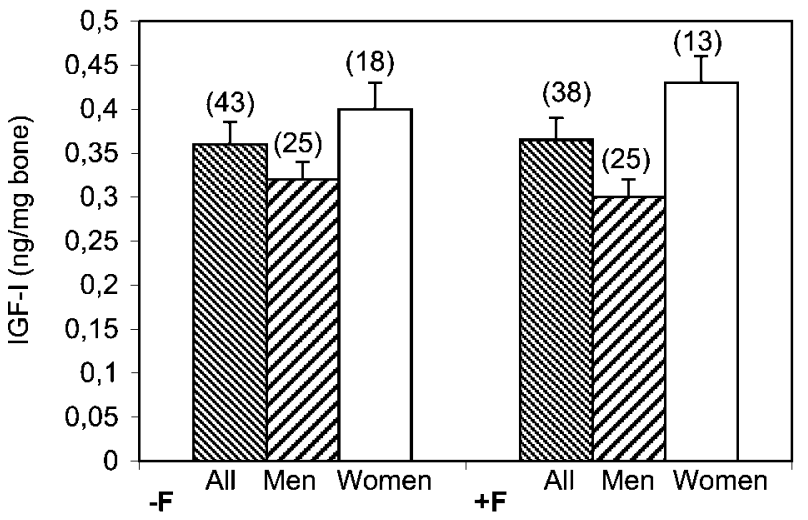

B

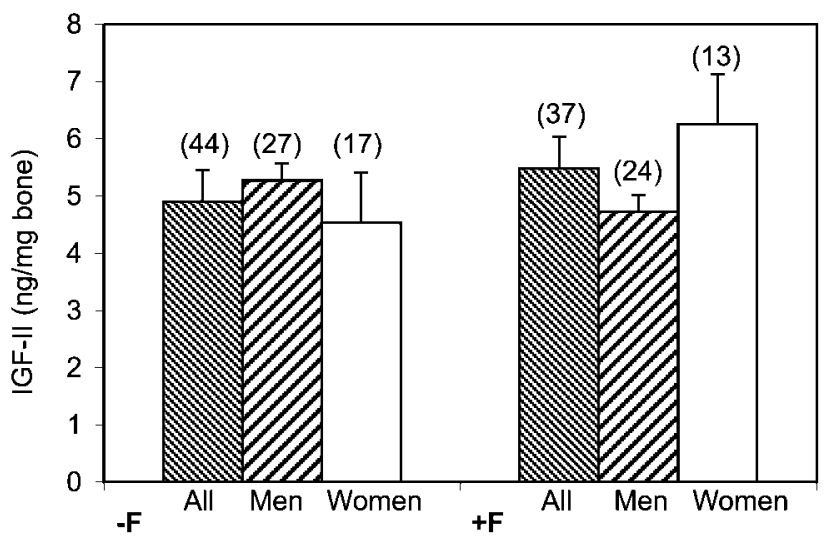

C

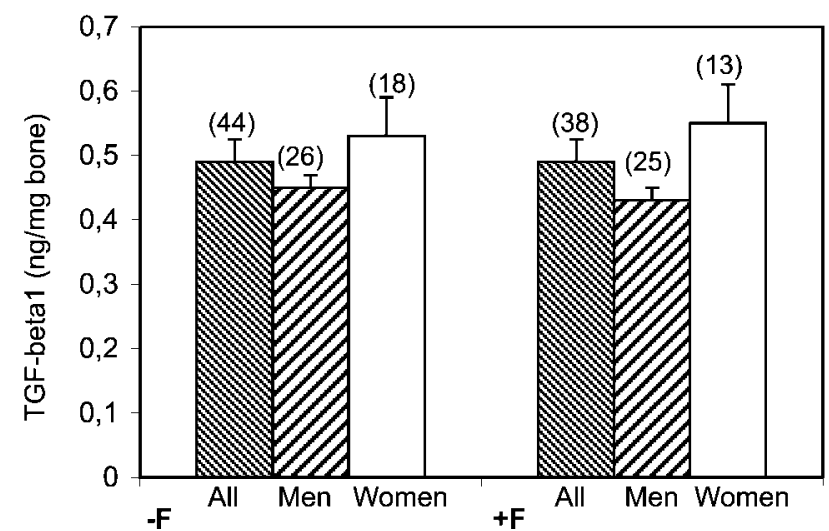

D

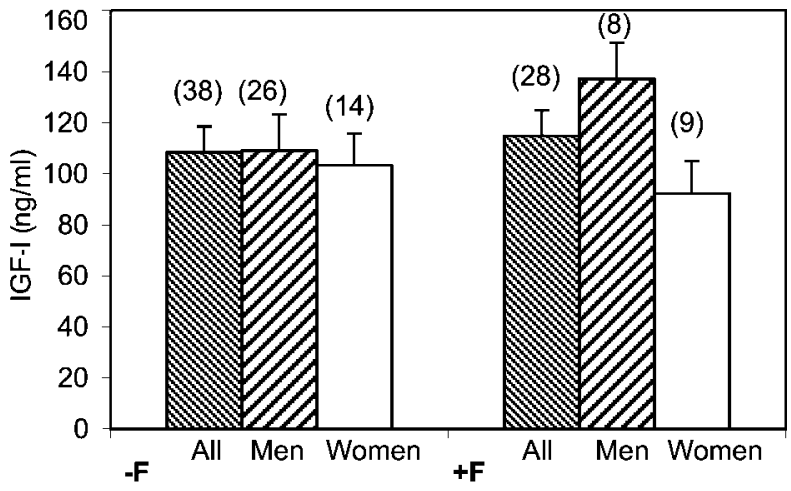

E

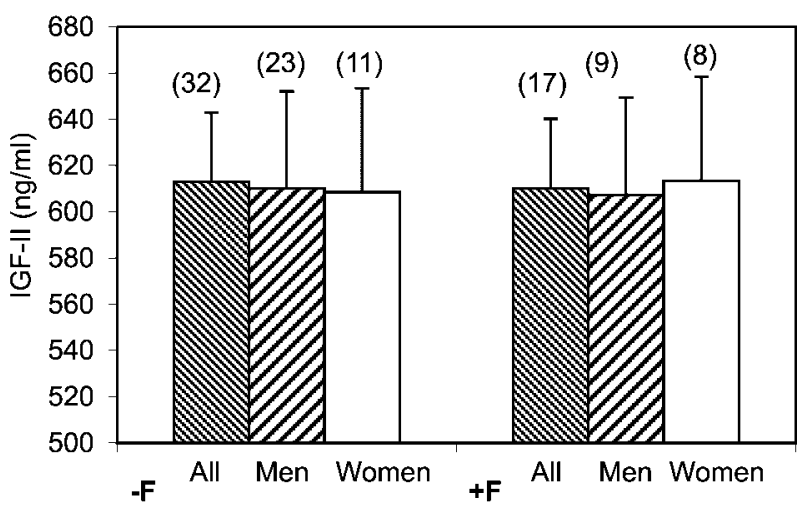

F

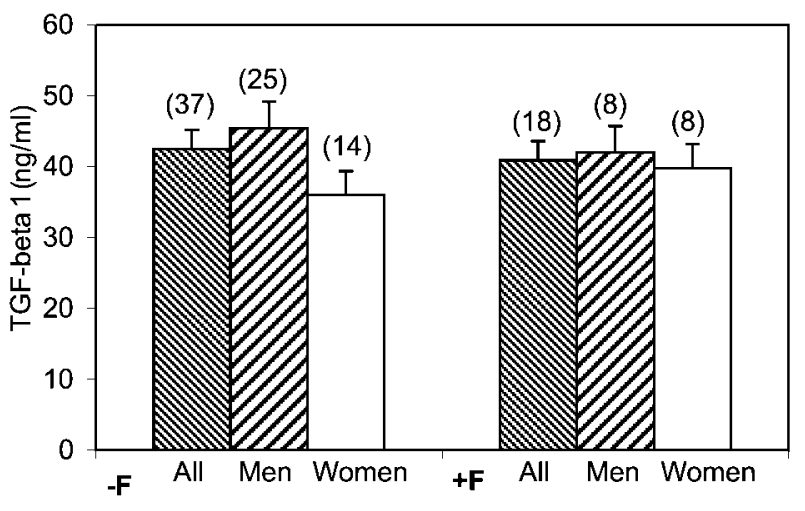

Figure 1 Effects of fluor therapy on the concentration of IGF-I, IGF-II and TGF- $\beta 1$ in bone matrix from patients with primary osteoporosis. The number of analyzed bone samples is shown in parentheses above each bar. Data are expressed as means \pm S.E.M. (A) Quantitation of IGF-I in bone matrix showed no difference between patients without fluor salt therapy $(-F)$ and age- and BMI-matched fluor salt therapy users $(+\mathrm{F})$, in either men $(P=0.83)$ or women $(P=0.82)$. (B) In multivariate analyses, no significant difference in bone matrix IGF-II concentration between non-users $(-\mathrm{F})$ and patients receiving fluor salt therapy $(+\mathrm{F})$ was noted, in either men $(P=0.56)$ or women $(P=0.27)$. (C) No significant difference in bone matrix TGF- $\beta 1$ concentration between non-users $(-F)$ and patients receiving fluor therapy $(+\mathrm{F})$ was noted, in either men $(P=0.62)$ or women $(P=0.46)$. (D) Fluor salt users had comparable levels of serum IGF-I to non-users (men: $P=0.23$, after adjustment for IGFBP-3, $P=0.06$; women: $P=0.51$, after adjustment for IGFBP-3, $P=0.20$ ).

(E) No significant difference in serum levels of IGF-II between patients receiving fluor ions therapy and controls was detected in either men $(P=0.95$, after adjustment for IGFBP-3, $P=0.86)$ or women $(P=0.93$, after adjustment for IGFBP-3, $P=0.45)$. (F) No significant difference in serum levels of TGF- $\beta 1$ between patients with fluor therapy and patients without fluor therapy was detected in either men $(P=0.43)$ or women $(P=0.38)$. 
A

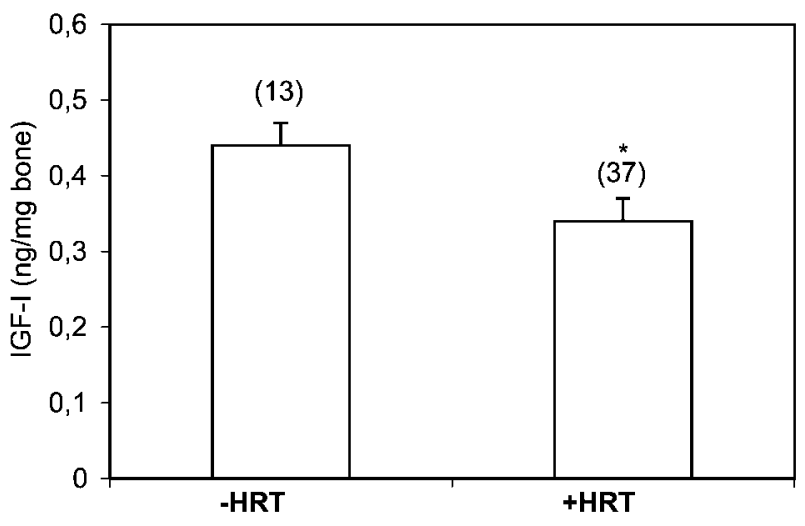

B

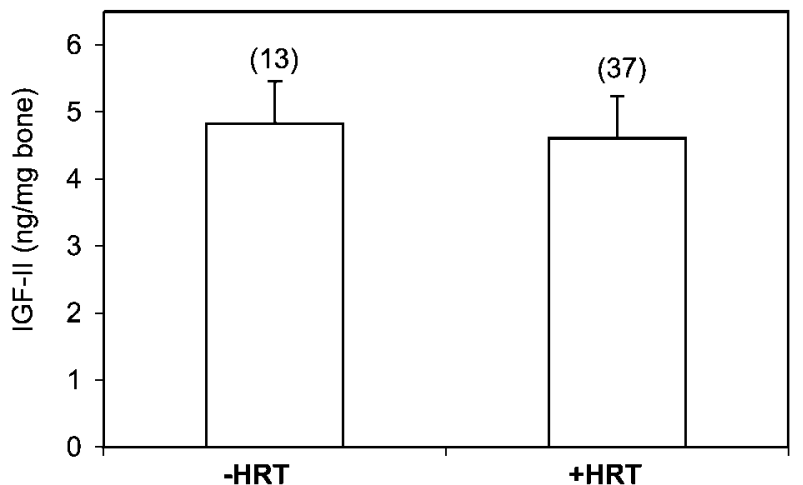

C

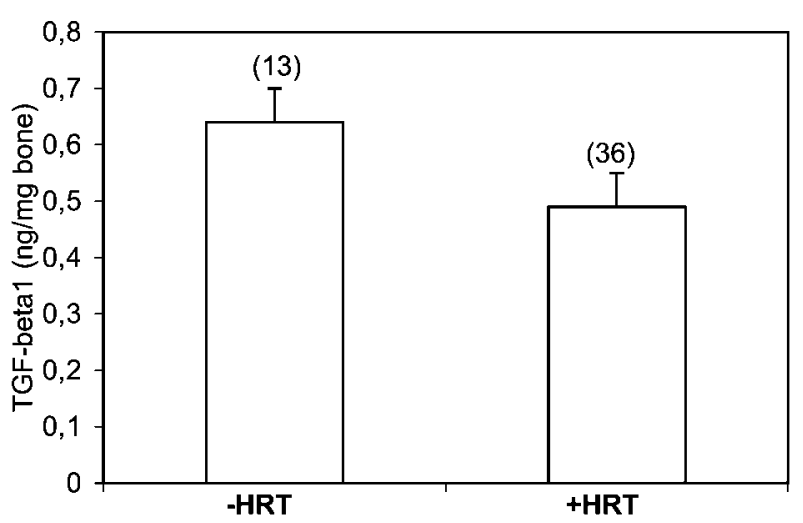

D

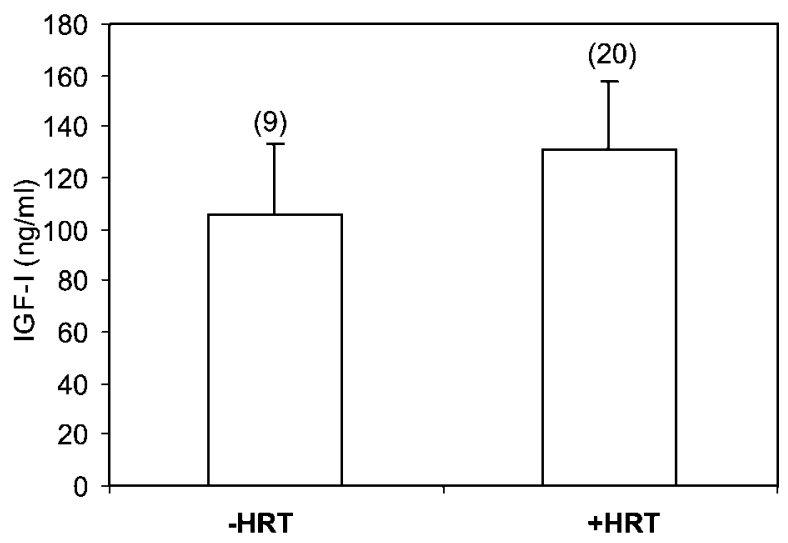

$\mathbf{E}$

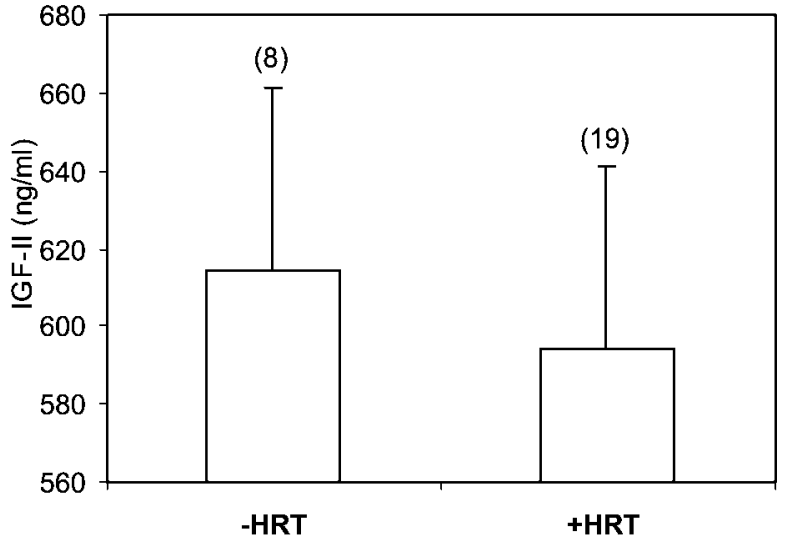

F

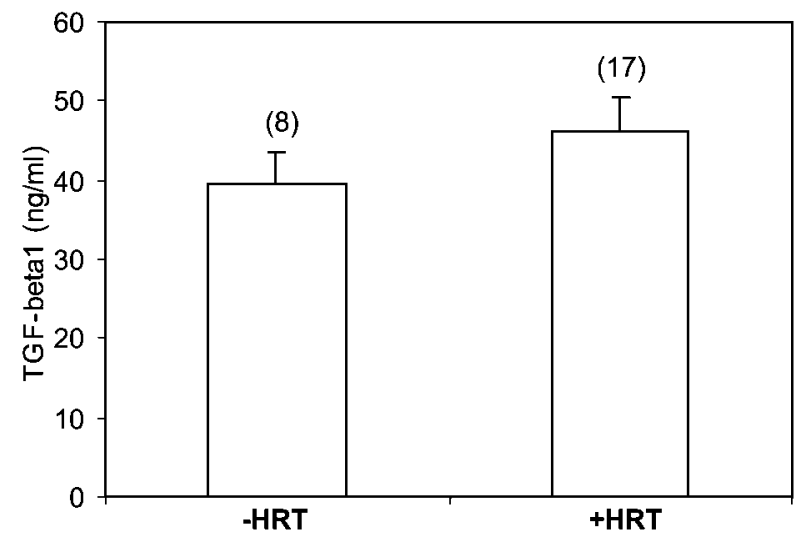

Figure 2 Effects of HRT on the concentration of IGF-I, IGF-II and TGF- $\beta 1$ in bone matrix from postmenopausal women with primary osteoporosis. The number of analyzed bone samples is shown in parentheses above each bar. Data are expressed as means \pm S.E.M. ${ }^{\star} P=0.03$. Postmenopausal women without HRT $(-\mathrm{HRT})$ had significantly higher IGF-I concentrations compared with age- and BMImatched women with HRT (+HRT) as shown in (A). However, this significance was lost after adjustment for serum AP levels $(P=0.20)$. (B) No significant difference in bone matrix IGF-II concentration between postmenopausal women on HRT and women without HRT was noted $(P=0.77)$. (C) There was a tendency for lower TGF- $\beta 1$ concentrations in postmenopausal women with HRT compared with women without HRT $(P=0.07)$ but this was lost in AP-adjusted analyses $(P=0.18)$. (D) No significant differences in serum IGF-I levels between postmenopausal women without HRT and with HRT was noticed $(P=0.41$, after adjustment for IGFBP-3 and concomitant therapy with fluor salts, $P=0.54$ ). (E) No significant differences in serum IGF-II levels between postmenopausal women without HRT and with HRT was observed $(P=0.73$, after adjustment for IGFBP-3 and concomitant therapy with fluor salts, $P=0.68)$. (F) No significant differences in serum TGF- $\beta 1$ levels between postmenopausal women without HRT and with HRT was noticed $(P=0.19$, after adjustment for concomitant fluoride therapy, $P=0.17)$. 
TGF- $\beta 1$ concentrations in bone matrix and the length of HRT $(n=38, r=-0.37, P=0.02)$ was noticed. No dependency of bone matrix growth factor levels with the dose of estrogens was observed in our group of patients (data not shown). Serum samples from patients receiving oral HRT for at least 12 months before bone biopsy $(n=29)$ were considered for comparison with age- and BMI-matched women without HRT $(n=11)$. No differences in serum concentrations of IGF-I, IGF-II or TGF- $\beta 1$ between groups were noticed (Fig. 2D, E and F), even after adjustment for IGFBP-3 was performed.

\section{Effects of calcitonin therapy on bone matrix and serum levels of IGF-I, IGF-II and TGF-B1 in patients with primary osteoporosis}

To evaluate whether or not calcitonin, a classic antiresorbtive drug, alters the concentration of skeletal growth factors, we compared the skeletal levels of these factors between two groups of patients with osteoporosis. A group of 16 subjects (10 men and 6 women) on calcitonin therapy (Table 1) was compared with 42 age- and BMI-matched controls (30 men and 12 women). Those patients and controls in whom calcitonin therapy was combined with HRT were excluded. Those in whom calcitonin was combined with fluor salts were not excluded but adjustment for this therapy was performed in multivariate analyses.

We observed no differences between groups in skeletal IGF-I or IGF-II levels (Fig. 3A and B). In addition, no significant difference in the concentration of bone matrix TGF- $\beta 1$ between calcitonin users and nonusers was detected (Fig. 3C). No association of skeletal growth factor levels with the length of calcitonin therapy was observed (men: IGF-I: $n=9, r=-0.51$, $P=0.18$; IGF-II: $n=9, r=-0.37, P=0.35$; TGF$\beta 1: \quad n=9, \quad r=-0.36, \quad P=0.37$; women: IGF-I: $n=5, r=0.31, P=0.68$; IGF-II: $n=5, r=-0.81$, $P=0.18$; TGF- $\beta 1: n=5, r=0.84, P=0.15)$. There was no change in the relationship between calcitonin therapy and the concentration of growth factors in bone matrix when the comparison was restricted to nine patients (six men and three women) receiving calcitonin for at least 12 months before bone biopsy and 24 age- and BMI-matched controls (18 men and six women) (data not shown). In multivariate analyses, serum IGF-I, IGF-II and TGF- $\beta 1$ levels were not different between subjects on continuous calcitonin therapy for more than 12 months and non-users (Fig. 3D, E and $\mathrm{F}$ ).

\section{Discussion}

The regulation of IGF and TGF- $\beta 1$ within the human skeleton is complex. In vitro data have shown that in human osteoblastic cell systems both IGF-I and TGF$\beta 1$ are under the control of estrogens and parathyroid hormone (PTH) $(9,10,24)$ and recently Farley et al. (12) have demonstrated stimulatory effects of calcitonin on IGF-I and IGF-II in human osteoblast-like cells. These data might lead us to speculate about the impact of these mechanisms on the regulation of normal human bone metabolism as well as during bone diseases. However, there is no evidence yet that these mechanisms are clearly relevant in vivo. Therefore, we have attempted to elucidate whether or not potential regulators of the skeletal content of IGF and TGF- $\beta 1$ may significantly influence the expression of these factors in vivo.

Bone is a dynamic tissue undergoing a lifelong remodeling process. Studies on bone metabolism have shown that cancellous bone matrix is renewed every $1-2$ years; therefore patients on long-term treatment with fluor salts, HRT or calcitonin were enrolled in the present study and compared with age- and BMI-matched controls in whom osteoporosis was not treated until the moment of bone biopsy.

While sodium fluoride acts by increasing bone formation in vitro (25) and in vivo, estrogens and calcitonin specifically act by inhibiting bone resorption. Fluoride has been shown to enhance the proliferative effect of IGF-I on osteoblastic cells in vitro (7). In the rat, sodium fluoride has been shown to potentiate the effect of IGF-I administration on BMD (26), suggesting an interaction between fluoride and the IGF system. In the present study, we were not able to detect significant differences in the concentration of IGF and TGF- $\beta 1$ in trabecular bone or in serum between fluor salt users and age- and BMI-matched non-users. Thus, it appears that mechanisms other than direct stimulation of IGF-I production by osteoblasts may explain the interaction between IGF-I and sodium fluoride. Indeed, in vitro studies have established that fluoride acts by increasing protein tyrosine phosphorylation (27), leading to the potentiation of osteoblast proliferation induced by growth factors.

In our group of postmenopausal women, HRT resulted in significantly lower bone matrix IGF-I but not IGF-II levels. This effect, however, was lost after adjustment of data for the rate of bone turnover. In the rat, only supra-physiological concentrations of $17 \beta$-estradiol were able to induce a stimulatory effect on the skeletal IGF-I level (28). In a previous report from our laboratory on the concentration of IGF-I and IGF-II in bone matrix from 533 women undergoing surgery for early stage breast cancer, menopausal transition appeared not to be associated with significant changes in trabecular bone matrix IGF-I or IGF-II levels (17). Our present findings are in accordance with the major anti-resorbtive effect of HRT as well as with previous data showing that IGF-I levels in the skeleton are highly related to bone turnover parameters (17). Differences in the rate of bone turnover were, as 
A

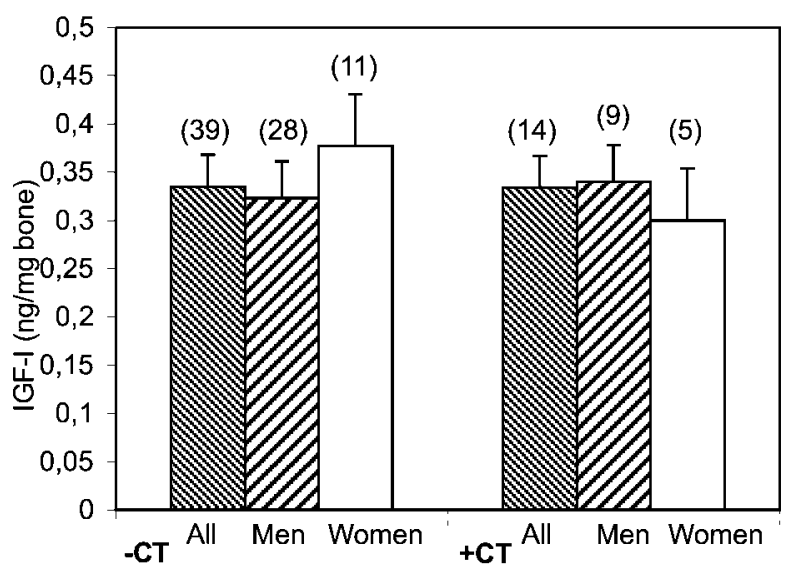

B

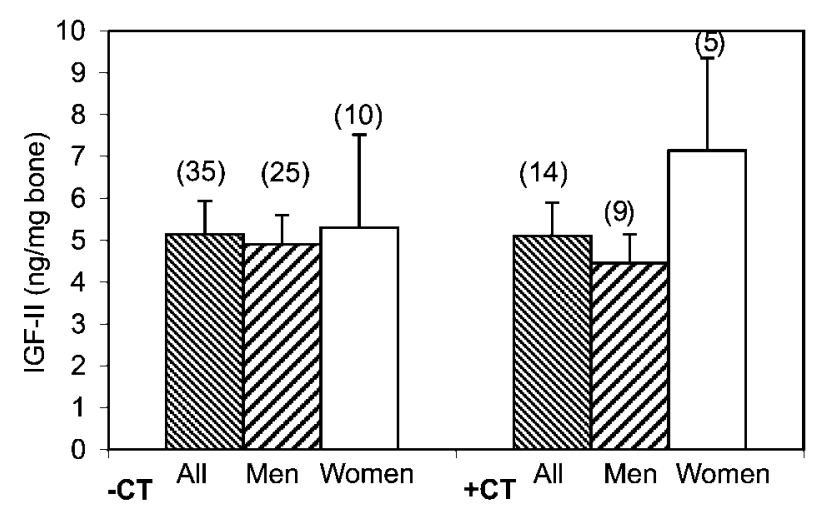

C

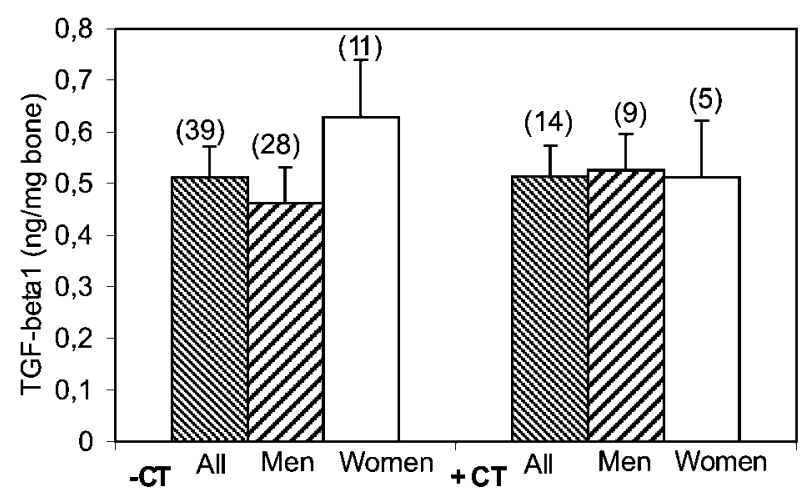

D

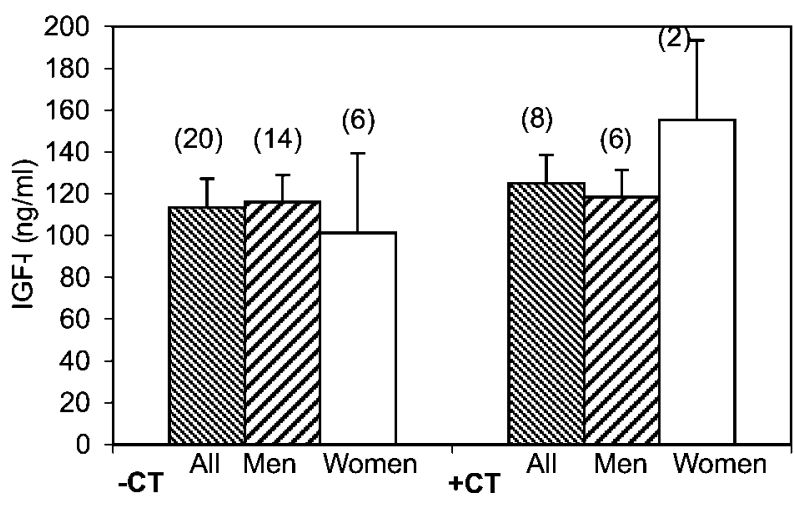

$\mathbf{E}$

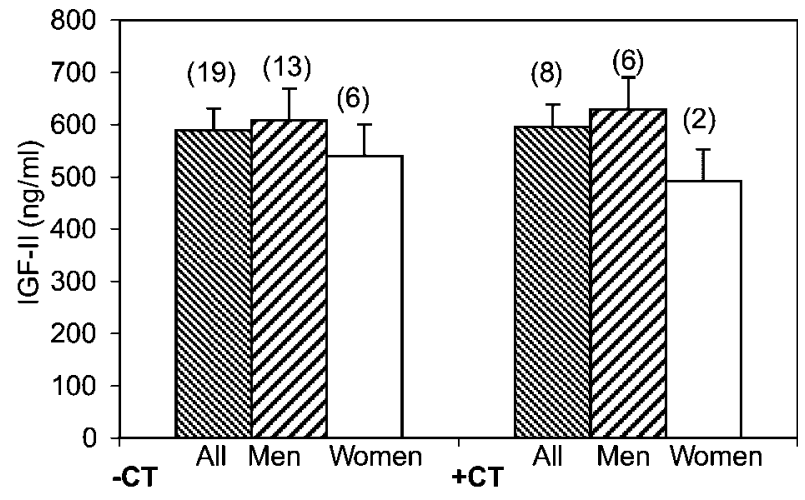

$\mathbf{F}$

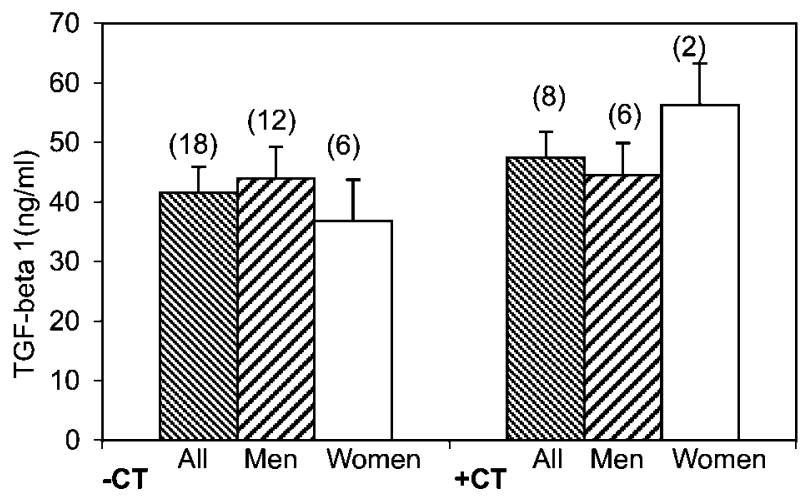

Figure 3 Effects of calcitonin on the concentration of IGF-I, IGF-II and TGF- $\beta 1$ in bone matrix from patients with primary osteoporosis. The number of analyzed bone samples is shown in parentheses above each bar. Data are expressed as means \pm S.E.M. (A) Measurements of IGF-I concentration in bone matrix showed no difference between patients on therapy with calcitonin ( $-\mathrm{CT})$ and age- and BMI-matched patients without calcitonin therapy $(+\mathrm{CT})$, in either men $(P=0.71)$ or women $(P=0.30)$. (B) No difference of bone matrix IGF-II in subjects with or without CT was observed, in either men $(P=0.60)$ or women $(P=0.56)$. (C) No difference of bone matrix TGF- $\beta 1$ between patients with and without CT was noticed, either in men $(P=0.054)$ or women $(P=0.42)$. (D) No significant difference in serum levels of IGF-I between patients with calcitonin and patients without calcitonin therapy was detected, in either men $(P=0.59$, after adjustment for IGFBP-3, $P=0.92)$ or women $(P=0.58$, after adjustment for IGFBP-3, $P=0.27)$. (E) Differences in serum levels of IGF-II between patients with calcitonin therapy and patients without calcitonin therapy were not significant, in either men $(P=0.76$, after adjustment for IGFBP-3, $P=0.68$ ) or women $(P=0.37$, after adjustment for IGFBP-3, $P=0.48)$. (F) No significant difference in serum TGF- $\beta 1$ levels between patients with calcitonin therapy and patients without calcitonin therapy was observed (men: $P=0.92$, women: $P=0.051$ ) 
well, responsible for variations in skeletal TGF- $\beta 1$ concentrations in the present study, with bone matrix levels of TGF- $\beta 1$ that tended to be lower in women with HRT as compared with women without HRT. These observations are in line with previous results from our laboratory showing that, in presumably healthy postmenopausal women, subjects on HRT have significantly lower skeletal TGF- $\beta 1$ levels in comparison with women not taking HRT (15).

Calcitonin has recently been shown to stimulate the production of IGF-I and IGF-II in human osteoblastic cells (12). In contrast, in our study, no significant effect of calcitonin on bone matrix and serum growth factor levels was seen. As some of our patients had received HRT concomitant with calcitonin, an interaction between estrogens and calcitonin with regard to their anti-resorbtive effects, as well as stimulatory effects of estrogen and progesterone on the endogenous calcitonin secretion $(29,30)$ in these women cannot be excluded. Thus, women receiving the combination of calcitonin and HRT were excluded from the analyses, despite lack of evidence that estrogens conserve bone by stimulating calcitonin secretion (31).

The $80 \%$ power of our study is sufficient to detect a meaningful biological difference in IGF-I concentrations between fluoride-treated patients and patients not treated with sodium fluoride of $30 \%$ and more. Similar calculations for each growth factor and each treatment group showed that differences of 30-35\% or more in bone matrix IGF-II and $40-55 \%$ or more in bone matrix TGF- $\beta 1$ concentrations, respectively, with fluoride, estrogens or calcitonin therapy could be well detected in this study.

Our previous studies have shown that $30 \%$ and more differences in IGF-I and TGF- $\beta$ concentrations in bone matrix occur with different states of bone metabolism. For example, large differences in IGF-I concentrations have been described with aging and after treatment with pharmacological agents such as PTH. A $36 \%$ reduction in IGF-I concentrations in human bone has been observed between the third and eighth decades of life (17). In the same study, a $20 \%$ higher bone volume was observed in women with bone matrix IGF-I concentrations in the upper range $(+2$ S.D. $)$ as compared with women with a bone matrix IGF-I concentration in the lower range $(-2$ s.D.). In comparison with vehicle-treated rats, a $30 \%$ increase in rat bone matrix IGF-I concentration and a $37 \%$ increase in rat bone matrix TGF- $\beta 1$ concentration was observed in PTH-treated animals (20). Changes in bone matrix growth factor concentrations to a similar extent may therefore have also been expected with fluoride treatment if fluoride has a strong impact on IGF-I bone matrix concentrations comparable with that of aging or PTH. Likewise, a 50\% decline in TGF- $\beta$ concentration in human bone has been observed between the third and eighth decades of life (15). In addition, there is evidence of a two- to threefold difference in
TGF- $\beta$ concentrations between bone samples of men and women with low bone turnover (1 quartile) as compared with high bone turnover (4 quartiles) (15). Nevertheless, it should be pointed out that smaller differences in growth factor concentrations might have been overlooked by our study.

There are some limitations to our study. Unfortunately, this is a cross-sectional study and the fact that unknown factors may have led to errors in the interpretation cannot be excluded. A longitudinal study, comparing the rate of change in the concentration of growth factor before and after therapy may be more sensitive to the effects of treatment. Secondly, some of the postmenopausal women in whom the effect of HRT on the IGF and TGF- $\beta 1$ concentration in bone matrix was assessed had received a combination of HRT and fluor salt therapy. On the other hand, we have shown here that there is no significant influence of fluor therapy on the bone matrix content of IGF and TGF- $\beta 1$. In addition, multivariate analysis was performed to adjust for concomitant therapy.

Both the control and the study group received supplements of calcium and vitamin D. Vitamin D on its own may affect bone metabolism; however, highly significant effects on osteoblastic cells and their growth factor production or regulation have been demonstrated especially for 1,25-dihydroxyvitamin D3 (32, 33). On the other hand, it should be pointed out that standard vitamin D supplements and not active metabolites of vitamin $\mathrm{D}$ were given to osteoporotic patients in this study.

Most data on the production of growth factors and their effects on bone cells has resulted from in vitro studies. Little is known about the relationship between growth factor production by bone cells, their release in the extracellular compartment and blood, and their integration in bone matrix. In addition, the timecourse of maximal concentrations of growth factors after the initiation of osteoporotic treatment is not known and is difficult to obtain in humans.

Despite in vitro data suggesting a role of skeletal IGFs and TGF- $\beta 1$ as mediators of estrogen and calcitonin action on bone metabolism, our study could not provide evidence of an in vivo relevance of this effects. In addition, fluor salts appeared not to influence the concentration of growth factors in the bone matrix of patient with primary osteoporosis directly.

\section{Acknowledgements}

We acknowledge the excellent technical assistance of Gisela Schwahn.

\section{References}

1 Canalis E. Skeletal growth factors and aging. Journal of Clinical Endocrinology and Metabolism 199478 1009-1010. 
2 Harris SE, Bonewald LF \& Harris MA. Effects of TGF-beta on bone nodule formation and expression of bone morphogenetic protein-2, osteocalcin, osteopontin, alkaline phosphatase and type I collagen in prolonged cultures of fetal rat calvarial osteoblasts. Journal of Bone and Mineral Research 19949 855-863.

3 Hock JM, Centrella M \& Canalis E. Insulin-like growth factor I (IGF-I) has independent effects on bone matrix formation and cell replication. Endocrinology 1988122 254-260.

4 Noda M \& Camilliere JJ. In vivo stimulation of bone formation by transforming growth factor $\beta$. Endocrinology $1989 \quad \mathbf{1 2 4}$ 2991-2994.

5 Mochizuki H, Hakeda Y, Waktsuki N, Usui N, Akashi S, Sato T et al. Insulin-like growth factor I supports formation and activation of osteoclasts. Endocrinology 1992131 1075-1080.

6 Farley JR, Tarbaux N, Murphy L, Masuda T \& Baylink DJ. In vitro evidence that bone formation may be coupled to resorption by release of mitogen(s) from resorbing bone. Metabolism 198736 314-321.

7 Lau KH, Farley JR, Freeman TK \& Baylink DJ. A proposed mechanism of the mitogenic action of fluoride on bone cells: inhibition of the activity of an osteoblastic acid phosphatase. Metabolism 198938 858-868.

8 Ernst M \& Rodan GA. Estradiol regulation of insulin-like growth factor-I expression in osteoblastic cells: evidence for a transcriptional control. Molecular Endocrinology 1991 5 1081-1089.

9 Kassem M, Okazaki R, Harris SA, Spelsberg TC, Conover CA \& Riggs BL. Estrogen effects on insulin-like growth factor gene expression in a human osteoblastic cell line with high levels of estrogen receptor. Calcified Tissue International $1998 \mathbf{6 2} 60-66$.

10 Oursler M, Cortese C, Keeting P, Anderson M, Bonde S, Riggs BL et al. Modulation of transforming growth factor-beta production in normal human osteoblast-like cells by 17 beta-estradiol and parathyroid hormone. Endocrinology 1991129 3313-3320.

11 Robinson JA \& Riggs BL. Osteoclasts and transforming growth factor $\beta$ : estrogen-mediated isoform-specific regulation of production. Journal of Clinical Endocrinology and Metabolism 1996 $137615-619$.

12 Farley J, Dimai HP, Stilt-Coffing B, Farley P, Pham T \& Mohan S. Calcitonin increases the concentration of insulin-like growth factors in serum-free cultures of human osteoblast-line cells. Calcified Tissue International $2000 \mathbf{6 7} 247-254$.

13 Nicolas V, Prewett A, Bettica P, Mohan S, Finkelman RD, Baylink DJ et al. Age-related decreases in insulin-like growth factor-I and transforming growth factor- $\beta$ in femoral cortical bone from both men and women: implications for bone loss with aging. Journal of Clinical Endocrinology and Metabolism $1994781011-1016$.

14 Nicolas V, Mohan S, Honda Y, Prewett A, Finkelman RD, Baylink DJ et al. An age-related decrease in the concentration of insulin-like growth factor binding protein-5 in human cortical bone. Calcified Tissue International 199557 206-212.

15 Pfeilschifter J, Diel I, Scheppach B, Bretz A, Krempien R, Erdmann J et al. Concentration of transforming growth factor beta in human bone tissue: relationship to age, menopause, bone turnover and bone volume. Journal of Bone and Mineral Research $199813716-730$.

16 Boonen S, Aersness J, Dequeker J, Nicholson P, Cheng X, Lowet G et al. Age-associated decline in human femoral neck cortical and trabecular content of insulin-like growth factor I: potential implications for age-related (type II) osteoporotic fracture occurrence. Calcified Tissue International 199761 173-178.

17 Seck T, Scheppach B, Scharla S, Diel I, Blum WF, Bismar H et al. Concentration of insulin-like growth factor (IGF)-I and -II in iliac crest bone matrix from pre- and postmenopausal women: relationship to age, menopause, bone turnover, bone volume, and circulating IGFs. Journal of Clinical Endocrinology and Metabolism 199883 2331-2337.
18 Seck T, Bretz A, Krempien R, Krempien B, Ziegler R \& Pfeilschifter J. Age-related changes in insulin-like growth factor I and II in human femoral cortical bone: lack of correlation with bone mass. Bone 199924 387-393.

19 Dequeker J, Pearson J, Reeve J, Henley M, Bright J, Felsenberg D et al. Dual X ray absorptiometry-cross-calibration and normative reference ranges for the spine: results of a European Community Concerted Action. Bone 199517 247-254.

20 Pfeilschifter J, Laukhuf F, Muller-Beckmann B, Blum WF, Pfister T \& Ziegler R. Parathyroid hormone increases the concentration of insulin-like growth factor-I and transforming growth factor beta 1 in rat bone. Journal of Clinical Investigation $199596767-774$.

21 Pfeilschifter J, Scheidt-Nave C, Leidig-Bruckner G, Woitge HW, Blum WE, Wüster C et al. Relationship between circulating insulin-like growth factor components and sex hormones in a population-based sample of 50- to 80-year-old men and women. Journal of Clinical Endocrinology and Metabolism $1996 \mathbf{8 1}$ 2534-2540.

22 Blum WF, Ranke MB \& Bierich JR. Radioimmunoassay for IGFs and IGFBPs. Growth Regulation 19941 (Suppl 1) 11-19.

23 Blum WF, Ranke MB \& Bierich JR. A specific radioimmunoassay for insulin-like growth factor II: the interference of IGF binding proteins can be blocked by excess IGF-I. Acta Endocrinologica 1988118 374-380.

24 Wu Y \& Kumar R. Parathyroid hormone regulates transforming growth factor beta 1 and beta 2 synthesis in osteoblasts via divergent signaling pathways. Journal of Bone and Mineral Research $200015879-884$.

25 Farley JR, Tarbaux NM, Vermeiden JP \& Baylink DJ. In vitro evidence that local and systemic skeletal effectors can regulate $\left[{ }^{3} \mathrm{H}\right]$-thymidine incorporation in chick calvarial cell cultures and modulate the stimulatory actions(s) of embryonic chick bone extract. Calcified Tissue International 198842 23-33.

26 Ammann P, Rizzoli R, Caverzasio J \& Bonjour JP. Fluoride potentiates the osteogenic effects of IGF-I in aged ovariectomized rats. Bone 199822 39-43.

27 Lau William KH \& Baylink DJ. Molecular mechanism of action of fluoride on bone cells. Journal of Bone and Mineral Research 1998 13 1660-1667.

28 Erdmann J, Storch S, Pfeilschifter J, Ochlich P, Ziegler R \& Bauss F. Effects of estrogen on the concentration of insulin-like growth factor-I in rat bone matrix. Bone 199822 503-507.

29 Greenberg C, Kukreja SC, Bowser EN, Hargis GK, Henderson WJ \& Williams GA. Effects of estradiol and progesterone on calcitonin secretion. Endocrinology 1986118 2594-2598.

30 Silver J \& Naveh-Many T. Calcitonin gene regulation in vivo. Hormone and Metabolic Research 199325 470-472.

31 Fletcher CD, Farish E, Leggate J \& Hart DM. Prevention of bone loss by hormone replacement therapy is probably not due to stimulation of calcitonin secretion. Acta Endocrinologica 1991 $124353-356$.

32 Price PA \& Baukol SA. 1,25-Dihydroxyvitamin D3 increases synthesis of the vitamin K-dependent bone protein by osteosarcoma cells. Journal of Biology and Chemistry $1980 \quad 255$ 11660-11663.

33 Kurose H, Yamaoka K, Okada S, Nakajima S \& Seino Y. 1,25Dihydroxyvitamin D3 $\left[1,25-(\mathrm{OH})_{2} \mathrm{D}_{3}\right]$ increases insulin-like growth factor I (IGF-I) receptors in clonal osteoblastic cells. Study on interaction of IGF-I and $1,25-(\mathrm{OH})_{2} \mathrm{D}_{3}$. Endocrinology $19901262088-2094$.

Received 26 March 2003

Accepted 2 October 2003 